\title{
CARATHÉODORY DISTANCE AND CONVEXITY WITH RESPECT TO BOUNDED HOLOMORPHIC FUNCTIONS
}

\author{
SU-SHING CHEN
}

\begin{abstract}
A characterization of connected complex manifolds with complete Carathéodory distances is obtained. Some basic facts concerning convexity of complex manifolds with respect to their algebras of bounded holomorphic functions are also given.
\end{abstract}

1. Introduction. In [4], S. Kobayashi has proved the following theorem which is a generalization of [3].

THEOREM 1. Let $X$ be a complex manifold with Carathéodory distance. Fix an arbitrary point $p$ of $X$ and let $F(p)$ be the set of bounded holomorphic functions $f$ on $X$ such that $f(p)=0$. If $X$ is complete with respect to the Carathéodory distance, then $X$ is convex with respect to $F(p)$ and hence is holomorphically convex.

The purpose of this paper is to prove the converse of Theorem 1 for connected complex manifolds. Thus a characterization of connected complex manifolds with complete Carathéodory distances may be obtained. Some basic facts concerning convexity of complex manifolds with respect to their algebras of bounded holomorphic functions are also given in this paper.

2. $B(X)$-convexity. Let $X$ be a complex manifold. We denote by $O(X)$ and $B(X)$ the algebra of holomorphic functions on $X$ and the algebra of bounded holomorphic functions on $X$ respectively. In this section, we give some basic facts concerning $B(X)$-convexity which is defined in the following. A complex manifold $X$ is said to be convex with respect to the algebra $B(X)$ (for brevity, $B(X)$-convex) if the set

$$
\hat{K}_{B}=\left\{p \in X|| f(p) \mid \leqq\|f\|_{K}, \text { for all } f \in B(X)\right\}
$$

is compact provided $K$ is a compact subset of $X$. A Siegel domain of the second kind is an example of domains in $C^{n}$ which are convex with respect to their algebras of bounded holomorphic functions [1]. We denote by

Received by the editors May 25, 1972 and, in revised form, October 17, 1972.

AMS (MOS) subject classifications (1970). Primary 32E10, 32H15, 32H20.

Key words and phrases. Carathéodory distance, Riemann domain, complex manifold, convexity with respect to bounded holomorphic functions.

(c) American Mathematical Society 1973 
$D$ a Riemann domain [2, p. 43] over $C^{n}$. From the following proposition we shall see that convexity with respect to bounded holomorphic functions occurs quite naturally.

Proposition 1. Let $D$ be a domain of holomorphy. Then $D=\cup_{k} D_{k}$, where $D_{k}$ is $B\left(D_{k}\right)$-convex and $\bar{D}_{k} \subset D_{k+1}$ for each $k$.

Proof. There is an increasing sequence $\left\{D_{k}\right\}$ such that $\bar{D}_{k} \subset D_{k+1}$ and $D_{k}$ is $O\left(D_{k+1}\right)$-convex for each $k$ [2, p. 283]. Since $D_{k}$ is relatively compact in $D_{k+1}$, the restriction $O\left(D_{k+1}\right) \mid D_{k}$ of $O\left(D_{k+1}\right)$ to $D_{k}$ is contained in $B\left(D_{k}\right)$. Therefore $D_{k}$ is $B\left(D_{k}\right)$-convex.

Proposition 2. Let $X_{1}$ and $X_{2}$ be two complex manifolds, $X_{2}$ be $B\left(X_{2}\right)$ convex and $\Phi$ be a proper holomorphic mapping from $X_{1}$ onto $X_{2}$. Then $X_{1}$ is $B\left(X_{1}\right)$-convex.

Proof. Let $K_{1}$ be a compact subset of $X_{1} . K_{2}=\Phi\left(K_{1}\right)$ is compact in $X_{2}$. Consequently $\left(\hat{K}_{2}\right)_{B}$ and $\Phi^{-1}\left(\left(\hat{K}_{2}\right)_{B}\right)$ are compact. Moreover $\left(\hat{K}_{1}\right)_{B} \subset$ $\Phi^{-1}\left(\left(\hat{K}_{2}\right)_{B}\right)$. Otherwise there is a point $p_{1} \in X_{1}-\Phi^{-1}\left(\left(\hat{K}_{2}\right)_{B}\right)$ and $\Phi\left(p_{1}\right)=$ $p_{2} \in X_{2}-\left(\hat{K}_{2}\right)_{B}$. There exists $f_{2} \in B\left(X_{2}\right)$ such that $\left|f_{2}\left(p_{2}\right)\right|>\left\|f_{2}\right\|_{K_{2}}$. Then $f_{2} \circ \Phi=f_{1} \in B\left(X_{1}\right)$ and $\left|f_{1}\left(p_{1}\right)\right|>\left\|f_{1}\right\|_{\Phi^{-1}\left(K_{2}\right)} \geqq\left\|f_{1}\right\|_{K_{1}}$. Hence $\left(\hat{K}_{1}\right)_{B}$ is compact.

3. Carathéodory distances on complex manifolds. Let $\Delta$ denote the open unit disk in $C$ and let $\rho$ denote the distance function on $\Delta$ defined by the Poincaré metric of $\Delta$. The Carathéodory pseudodistance $c$ of a complex manifold $X$ is defined by

$$
c(p, q)=\sup \rho(f(p), f(q)) \text { for } p, q \in X,
$$

where the supremum is taken with respect to the family of all holomorphic mappings $f: X \rightarrow \Delta$. $A$ complex manifold $X$ is said to be $B(X)$-separable if the algebra $B(X)$ separates points of $X$. The necessary and sufficient condition for the Carathéodory pseudodistance $c$ to be a distance on $X$ is that $X$ is $B(X)$-separable. A manifold $X$ is complete with respect to the Carathéodory distance if for each point $p \in X$ and each positive number $r$ the closed ball of radius $r$ around $p$ is a compact subset of $X$. Fix a point $p \in X$. Let $F(p)$ be the set of all bounded holomorphic functions $f$ on $X$ such that $f(p)=0$. The following theorem is the converse of Theorem 1 for connected complex manifolds.

THEOREM 2. Let $X$ be a connected complex manifold. If $X$ is $F(p)-$ convex for some $p$ in $X$ and $X$ is $B(X)$-separable, then $X$ is complete with respect to the Carathéodory distance on $X .^{1}$

\footnotetext{
${ }^{1}$ The author is indebted to Professor S. Kobayashi for this improved statement.
} 
Proof. Consider the closed ball $\bar{B}(a, p)=\{q \in X \mid c(p, q) \leqq a\}$ with center $p \in X$ and radius $a>0$. We have to prove that $\bar{B}(a, p)$ is compact. Denote by $F_{+}(p)$ the set of all holomorphic mappings $f: X \rightarrow \Delta$ of $X$ into $\Delta$ such that $f(p)=0$ and $\|f\|_{X}=\sup _{q \in X}|f(q)|=1$. We claim that the convex hulls $\hat{K}_{F(p)}$ and $\hat{K}_{F_{+}(p)}$ coincide. It is obvious that $\hat{K}_{F(p)} \subset \hat{K}_{F_{+}(p)}$. For each $f$ in $F(p), g=f /\|f\|$ is in $F_{+}(p)$. Each point $q \in \hat{K}_{F_{+}(p)}$ satisfies $|g(q)| \leqq$ $\|g\|_{K}$ and hence $|f(q)| \leqq\|f\|_{K}$. Therefore $\hat{K}_{F_{+}(p)} \subset \hat{K}_{F(p)}^{+}$and $X$ is $F(p)$ convex if and only if it is $F_{+}(p)$-convex. Now it is sufficient to prove $\bar{B}(a, p)$ to be compact when $X$ is $F_{+}(p)$-convex. For each $f \in F_{+}(p)$ and each $q \in \bar{B}(a, p)$, we have

$$
\begin{gathered}
\rho(0, f(q)) \leqq c(p, q) \leqq a, \\
\frac{1}{2} \log \frac{1+|f(q)|}{1-|f(q)|} \leqq a \text { and }|f(q)| \leqq \frac{e^{2 a}-1}{e^{2 a}+1} .
\end{gathered}
$$

Being locally compact and second countable, ${ }^{2} X=\bigcup_{k} X_{k}$, where $X_{k} \subset X_{k+1}$ and $X_{k}$ is compact in $X$ for each $k$. There is a nondecreasing sequence $\left\{\inf _{F_{+}(p)}\|f\|_{X_{k}}\right\}$ of real numbers approaching to 1 as the limit when $k \rightarrow \infty$. For each real number $b, 0<b<1$, we can find a sufficiently large $k(b)$ such that for $k>k(b), \inf _{F_{+}(p)}\|f\|_{X_{k}}>b$. Consequently for each $q \in \bar{B}(a, p)$ and $f \in F_{+}(p)$ we have

$$
|f(q)| \leqq \frac{e^{2 a}-1}{e^{2 a}+1}=b<\|f\|_{X_{k}} \quad \text { (for some } k \text { ). }
$$

This implies that $\bar{B}(a, p) \subset\left(\hat{X}_{k}\right)_{F_{+}(p)}$. Since $X$ is $F_{+}(p)$-convex and $\bar{B}(a, p)$ is closed, $\bar{B}(a, p)$ is compact in $X$.

Corollary. A connected manifold $X$ is complete with respect to its Carathéodory distance if and only if $X$ is $F(p)$-convex for some $p$ in $X$ and $B(X)$-separable.

\section{REFERENCES}

1. S. Chen, Bounded holomorphic functions in Siegel domains (to appear).

2. R. C. Gunning and H. Rossi, Analytic functions of several complex variables, Prentice-Hall, Englewood Cliffs, N.J., 1965. MR 31 \#4927.

3. H. Horstmann, Carathéodorysche Metrik und Regularitätshullen, Math. Ann. 108 (1933), 208-217.

4. S. Kobayashi, Hyperbolic manifolds and holomorphic mappings, Pure and Appl. Math., no. 2, Dekker, New York, 1970. MR 43 \#3503.

5. K. Stein, Überlagerungen holomorph-vollständiger komplexer Räume, Arch. Math. 7 (1956), 354-361. MR 18, 933.

Department of Mathematics, University of Florida, Gainesville, Florida 32601

${ }^{2}$ A complex manifold is assumed to be paracompact. 